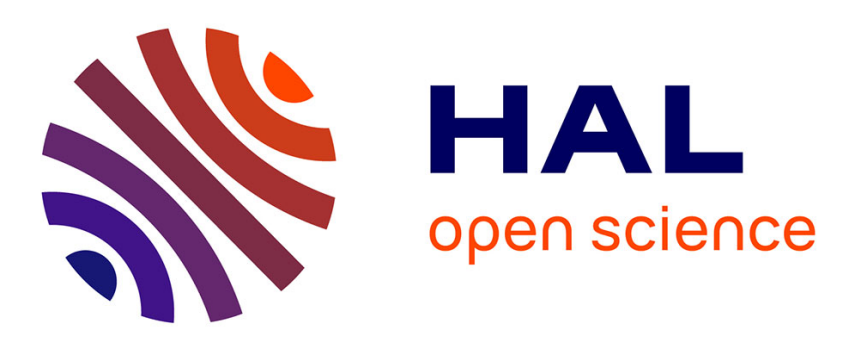

\title{
A Generalized Direction in Interior Point Method for Monotone Linear Complementarity Problems
}

\author{
Mounir Haddou, Tangi Migot, Jérémy Omer
}

\section{To cite this version:}

Mounir Haddou, Tangi Migot, Jérémy Omer. A Generalized Direction in Interior Point Method for Monotone Linear Complementarity Problems. Optimization Letters, 2019, 13 (1), pp.35-53. 10.1007/s11590-018-1241-2 . hal-01355566

\section{HAL Id: hal-01355566 https://hal.science/hal-01355566}

Submitted on 23 Aug 2016

HAL is a multi-disciplinary open access archive for the deposit and dissemination of scientific research documents, whether they are published or not. The documents may come from teaching and research institutions in France or abroad, or from public or private research centers.
L'archive ouverte pluridisciplinaire HAL, est destinée au dépôt et à la diffusion de documents scientifiques de niveau recherche, publiés ou non, émanant des établissements d'enseignement et de recherche français ou étrangers, des laboratoires publics ou privés. 


\title{
A Generalized Direction in Interior Point Method for Monotone Linear Complementarity Problems
}

\author{
M.Haddou, T.Migot, J.Omer
}

August 23, 2016

\begin{abstract}
In this paper, we present a new interior point method with full Newton step for monotone linear complementarity problems. The specificity of our method is to compute the Newton step using a modified system similar to that introduced by Darvay in [11. We prove that this new method possesses the best known upper bound complexity for these methods. Moreover, we extend results known in the literature since we consider a general family of smooth concave functions in the Newton system instead of the square root. Some computational results are included to illustrate the validity of the proposed algorithm.

Keywords : complementarity, concave functions, interior-point methods, linear programming, linear complementarity problems, polynomial time complexity

Mathematics Subject Classification : 49M05 - 65K15 - 90C33 $90 \mathrm{C51}$
\end{abstract}

\section{Notations}

Through this paper we will use the following notations: $\mathbb{R}_{+}^{n}=\left\{x \in \mathbb{R}^{n} \mid x \geq 0\right\}$, $\mathbb{R}_{++}^{n}=\left\{x \in \mathbb{R}^{n} \mid x>0\right\}$ and $\mathbf{e}$ denotes the vector with all entries equal to one and whose dimension can be deduced from the context. Given two vectors $z, s \in \mathbb{R}^{n}$, we denote by $z^{T} s$ the usual scalar product and by $z s$ the Hadamard product of two vectors, that is $z s=\left(z_{i} s_{i}\right)_{1 \leq i \leq n}$. Moreover, we extend this component-wise operation to the division of two vectors and to the square root, that is $z / s=\left(z_{i} / s_{i}\right)_{1 \leq i \leq n}$ and $\sqrt{z}=\left(\sqrt{z_{i}}\right)_{1 \leq i \leq n}$.

\section{Introduction}

Since the early 80's and Karkamar's method [18, interior point methods (IPMs) have received a wide interest in the literature, in several monographs [19, 24, 
[27, 28, 30] or $\mathrm{PhD}$ thesis [13, 14, 21, to cite only a few of them. This enthusiasm for IPMs mainly comes from their theoretical complexity. Indeed, large families of problems including linear programs (LPs) and convex quadratic programs (CQPs) that are special cases of linear complementarity problems (LCPs) can be solved in polynomial time.

An LCP consists in finding $z, s \in \mathbb{R}^{n}$ such that for a square matrix $M \in \mathbb{R}^{n \times n}$ and a vector $q \in \mathbb{R}^{n}$

$$
s=M z+q, z, s \geq 0, z s=0 .
$$

A couple $(z, s)$ such that $s=M z+q$ is said to be feasible for the LCP if we have $z, s \geq 0$ and strictly feasible if $z, s>0$. From now on, we use standard notation $\mathcal{F}_{+}:=\left\{(z, s) \in \mathbb{R}_{++}^{2 n} \mid s=M z+q\right\}$ for the set of strictly feasible points of ( $\mathrm{LCP})$.

The main strategy of IPMs is to follow the central path $(z(\boldsymbol{\mu}), s(\boldsymbol{\mu}))$ for $\boldsymbol{\mu} \in \mathbb{R}_{++}^{n}$, defined by

$$
s=M z+q, z, s \geq 0, z s=\boldsymbol{\mu} .
$$

The couples $(z(\boldsymbol{\mu}), s(\boldsymbol{\mu}))$ are also called $\boldsymbol{\mu}$-centers and define an homotopic path. The limit when $\boldsymbol{\mu} \rightarrow 0$ satisfies the complementarity condition, and hence yields optimal solutions whenever the limit exists.

IPMs follow the central path approximately by solving an approached version of the non-linear system in $\left(\overline{\mathrm{LCP}_{\mu}}\right)$ for several values of $\boldsymbol{\mu}$. The main tool to solve such a system is the Newton method. A Newton step $(\Delta z, \Delta s)$ is given as the solution of the following linear system

$$
\left\{\begin{array}{l}
M \Delta z=\Delta s \\
z \Delta s+s \Delta z=\boldsymbol{\mu}-z s
\end{array} .\right.
$$

There exists a wide variety of different IPMs that are based on this principle. In this paper we focus on the simplest IPM (see Algorithm 1) : the full Newton step interior point method (FN-IPM). Despite its simplicity, this method is one among the IPMs with the best worst-case complexity. Therefore, it is natural that any new try in the IPM framework must be validated on this method.

In his monograph [11], Darvay introduces a modification in $\left(\overline{\mathrm{LCP}_{\mu}}\right)$ by considering

$$
s=M z+q, z, s \geq 0, \varphi(z s)=\boldsymbol{\mu},
$$

where $\varphi: \mathbb{R}_{+}^{n} \rightarrow \mathbb{R}_{+}^{n}$ is assumed to be a smooth function such that $\varphi(0)=0$ and $\varphi$ is defined by a component-wise extension of a real-valued function $\varphi$ to $\mathbb{R}^{n}$, i.e for $t \in \mathbb{R}^{n} \varphi(t)=\left(\varphi\left(t_{i}\right)\right)_{1 \leq i \leq n}$.

This new direction has become an active subject in the past few years for $\varphi(t)=\sqrt{t}$. For instance, the original result that Darvay established for linear programming is extended to convex quadratic programming and monotone LCP in [1, 2]. Furthermore in [4, 29, the authors extend the result of worst-case polynomial complexity to the wider class of sufficient LCPs for FN-IPM. Finally 


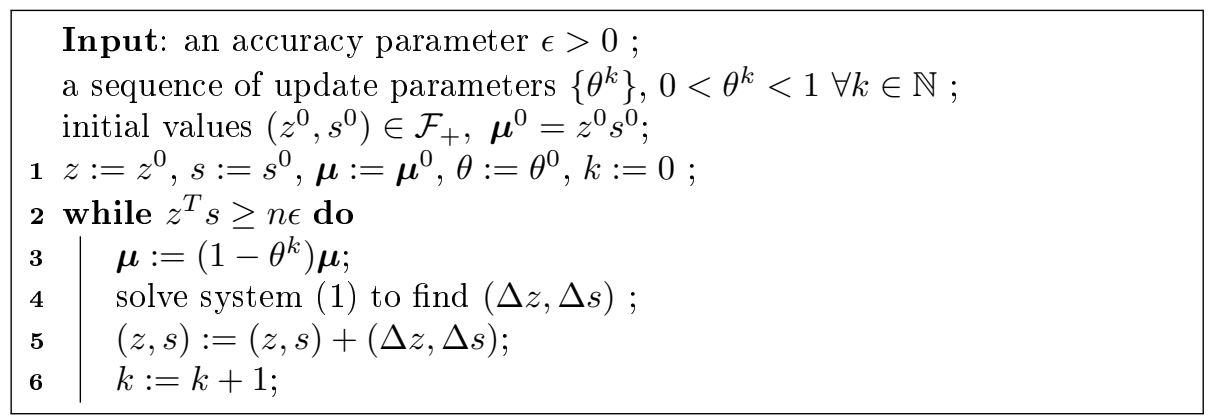

Algorithm 1: Full Newton step IPM (FN-IPM)

a method independent of the choice of the initial iterate can be found in $[5$. In all these different contexts, this new technique with $\varphi(t)=\sqrt{t}$ gives the best known complexity upper bound. This motivates our goal to study a larger family of functions.

Darvay's method modifies the Newton steps. More precisely, the Newton step $(\Delta z, \Delta s)$ is given by the linear system

$$
\left\{\begin{array}{l}
M \Delta z=\Delta s \\
\varphi^{\prime}(z s)(z \Delta s+s \Delta z)=\boldsymbol{\mu}-\boldsymbol{\varphi}(z s)
\end{array}\right.
$$

In this paper we consider functions $\varphi \in C^{3}\left(\mathbb{R}_{+}\right)$such that $\varphi(0)=0, \varphi$ is increasing and concave, and $\varphi^{\prime \prime \prime}(t) \geq 0 \forall t \in \mathbb{R}_{+}$. This class of functions allows for a generalisation of the classical IPMs, since we obtain the classical central path system $\left(\mathrm{LCP}_{\mu}\right)$ for $\varphi(t)=t$. The square root function does not belong to this family since it is not differentiable at 0 , however we can consider a smooth version of the square root with $\varphi_{\epsilon>0}: t \mapsto \sqrt{t+\epsilon}-\sqrt{\epsilon}$. Algorithm 1 can then be modified to solve (3) instead of (1) at step 4. We call the resulting algorithm $\varphi$-FN-IPM. The main result of this article is that $\varphi$-FN-IPM, converges to an $\epsilon$-solution in at most

$$
\mathcal{O}\left(\sqrt{n} \log \left(\frac{n}{\epsilon}\right)\right)
$$

iterations. This upper bound corresponds to the best known upper bound for the FN-IPM.

In Section 2, we present the state of the art of LCPs and IPMs. In Section 3, we show the polynomial compexity of $\varphi$-FN-IPM. Finally, in Section 4 , we provide some numerical results on specific examples to illustrate the validity of the proposed algorithm. 


\section{State of the Art}

\subsection{The Linear Complementarity Problem}

The $(\overline{\mathrm{LCP}})$ is a feasibility and not an optimization problem, but it is wellknown that it is closely related with optimization problems. Indeed optimality conditions of some optimization problems such as LPs and QPs can be stated as LCPs.

For general matrices $M$, the problem is NP-complete 8 . Therefore, it is natural to look for classes of matrices $M$ for which the corresponding LCPs can be solved in polynomial time. There are more than 50 matrix classes discussed in the literature of LCPs and a survey can be found in [9. Below we list several important and frequently used classes in an IPM framework :

- $P_{*}(\kappa)$-matrices : for $\kappa \geq 0$ the following property holds :

$$
\forall x \in \mathbb{R}^{n}(1+4 \kappa) \sum_{i \in \mathcal{I}^{+}(x)} x_{i}(M x)_{i}+\sum_{i \in \mathcal{I}^{-}(x)} x_{i}(M x)_{i} \geq 0,
$$

where $\mathcal{I}^{+}(x)=\left\{i: x_{i}(M x)_{i}>0\right\}$ and $\mathcal{I}^{-}(x)=\left\{i: x_{i}(M x)_{i}<0\right\}$;

- $P_{*}=\cup_{\kappa \geq 0} P_{*}(\kappa)$;

- skew-symmetric matrices : $\forall x \in \mathbb{R}^{n}: x^{T} M x=0$;

- positive semidefinite (PSD) matrices : $\forall x \in \mathbb{R}^{n}: x^{T} M x \geq 0$;

- $P_{0}$-matrices : matrices with all principal minors non-negative, i.e $\forall x \neq 0 \in \mathbb{R}^{n}: \exists i, x_{i}(M x)_{i} \geq 0$.

The most common and most studied class is that of monotone LCPs, where $M$ is a positive semidefinite matrix. In the special case of the optimality conditions of an LP, $M$ is a skew-symmetric matrix. The class of $P_{*}$-matrices was introduced by Kojima et al. in their fundamental monograph on IPMs for LCPs [19], while the other classes, as well as a number of additional classes not mentioned here, were discussed in the classical monograph of Cottle, Pang, and Stone [10] and in [3]. Most of the relations between these classes are described in Figure1. Some relations are trivial such that the positive semidefinite matrices $\left(=P_{*}(0)\right)$ are in $P_{*}$ whereas others are not.

It is well known that the sequence of the iterates of an IPM may not converge to a solution of an LCP. To ensure that each limit point of the sequence of the iterates is a solution, the sequence must be bounded. The following question arises: Which class of matrices implies boundedness of the sequence? In [19], Kojima et al. present an IPM for LCPs and proved its global convergence under the following conditions [Condition 2.3, [19]] :

(i) $M$ is a $P_{0}$-matrix;

(ii) there exists a strictly feasible point $\left(z^{0}, s^{0}\right)>0$ and $s^{0}=M z^{0}+q$. 


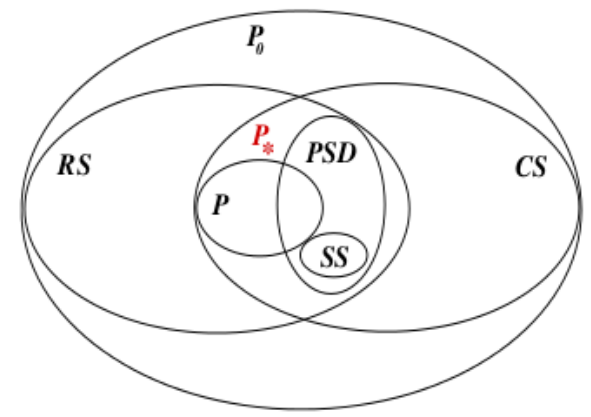

Figure 1: The inclusion relations between various matrix classes; $\mathrm{CS}=$ column sufficient, RS =row sufficient, SS = skew-symmetric, PSD = positive semidefinite. Source : 12

(iii) the level set $\left\{(z, s) \in \mathbb{R}_{++}^{2 n} \mid s=M z+q, z^{T} s \leq t\right\}$ is bounded for each $t \geq 0$.

Conditions (i) and (ii) guarantee existence and uniqueness of the central path. Note that condition (ii) is sometimes called "interior point condition" in the literature.

The linear systems (1) and (3) admit a unique solution as a consequence of condition (i) as stated in the following proposition.

Proposition 2.1 ([19]). $M$ is a $\mathcal{P}_{0}$-matrix if and only if $\left(\begin{array}{cc}M & -I \\ S & Z\end{array}\right)$ is a nonsingular matrix. (for any positive diagonal matrices $Z, S$ )

Kojima et al. point out that, unfortunately, if $M$ is only a $P_{0}$-matrix, then requirement (iii) is in general not satisfied, and they provide a counterexample. However, if $M$ is a $P_{*}$-matrix, then requirement (iii) is satisfied (Lemma 4.5 in [19]). Hence, in some sense the $P_{*}$-class is maximal with respect to the property that guarantees global convergence of IPMs.

In this paper we consider a monotone linear complementarity problem, i.e an LCP where $M$ is PSD. In this case the set of solution of $(\overline{\mathrm{LCP}})$ is a convex set.

\subsection{Interior Point Methods}

There exists a wide variety of IPMs that approximately follow the central path. They are sometimes called path-following-methods in the literature. These methods go through three main steps: find an initial point, compute some Newton steps (possibly some damped Newton steps) and update the parameter $\boldsymbol{\mu}$.

In this paper, we focus on the most simple among these methods: the FNIPM. This method is described in Algorithm 1 and consists in taking a single 
full Newton step for each $\boldsymbol{\mu}$. In general, the value of the update parameter $\theta$ is an $\mathcal{O}(1 / \sqrt{n})$. Since the Newton method with full step behaves well locally it is not a surprise that the iterates generated by this method are close to the central path. More informations about FN-IPM can be found in the monographs 27. for LPs or in [30] for a more general setting. One can also consider an adaptive update version of the algorithm, which tries to find the best value of $\theta$ at each iteration, see [17, 27. Therefore, a sequence of $\theta$ is used instead of one fixed value. Worst-case complexity for these methods is in $\mathcal{O}(\sqrt{n} \log (n / \epsilon))$, which is the best result known for IPMs. However they do not perform well in practice, because the iterates stay too close to the central path.

In some sense a dual approach is to first decide a fixed value of $\theta \in] 0,1[$. With this approach, the iterates are more likely to be far from the central path, so it is necessary to carry out some damped Newton steps with a damping parameter $\alpha$ to bring the iterates closer to the central path. By taking $\theta=\mathcal{O}(1 / \sqrt{n})$ we get the FN-IPM, since it is probably not necessary to use more than one centralization step. This leads to what is sometimes called in the literature the small-update IPM. However by taking $\theta=\mathcal{O}(1)$ we get the large-update method. In order to determine the value of the damping parameter $\alpha$ some merit function is used. The first among the merit functions is the logarithmic barrier, 27. More recently Peng, Roos and Terlaky consider self-concordant barrier [23] and kernel functions [13, 7, 20]. Worst-case complexity for these methods to find an $\epsilon$-solution to ( $\mathrm{LCP})$ is in $\mathcal{O}(\sqrt{n} \log (n) \log (n / \epsilon))$. This result is quite poor compared to small-update methods, in particular if we consider that the large update methods behave much better in practice. This gap between theory and practice is called "irony of IPMs" in [24] and is a main motivation to try modified versions of these methods.

Note that in all the methods mentioned above, a strictly feasible initial point is assumed to be known. In many cases, finding such point is not trivial. Two approaches allow to do this. The first one is a self-embedding technique, which enlarges the problem to create an artificial initial point on the central path. This technique is presented in [27] for LPs and in [19] for general LCPs. A second technique is to consider an infeasible version of the IPM. Given an initial point $\left(z^{0}, s^{0}\right) \in \mathbb{R}_{++}^{2 n}$ with $z^{0} s^{0}=\boldsymbol{\mu}^{0}$, which does not satisfy $s=M z+q$ we solve the following system

$$
s-M z-q=\frac{\boldsymbol{\mu}}{\boldsymbol{\mu}^{0}}\left(s^{0}-M z^{0}-q\right), z, s \geq 0, z s=\boldsymbol{\mu} .
$$

This method gives asymptotically feasible iterates. Such methods are considered in [5, 6, 22, 26].

\section{Polynomial complexity}

In this section, we consider the worst-case complexity of the $\varphi$-FN-IPM described in Algorithm 2 with $\varphi: \mathbb{R}_{+} \rightarrow \mathbb{R}_{+}$verifying

(i) $\varphi(0)=0$; 
(ii) $\varphi \in C^{3}([0,+\infty))$;

(iv) $\varphi^{\prime}(t)>0, \forall t \geq 0$, i.e $\varphi$ is increasing;

(v) $\varphi^{\prime \prime}(t) \leq 0, \forall t \geq 0$, i.e $\varphi$ is concave;

(vi) $\varphi^{\prime \prime \prime} \geq 0$.

These functions are obviously invertible and can be extended in a smooth way for negative $t$, for instance by considering : $\varphi(t<0)=t \varphi^{\prime}(0)+\frac{t^{2}}{2} \varphi^{\prime \prime}(0)+\frac{t^{3}}{6} \varphi^{\prime \prime \prime}(0)$. Function $\varphi: \mathbb{R}^{n} \mapsto \mathbb{R}^{n}$ is then defined component-wisely: $\forall t \in \mathbb{R}^{n}, \boldsymbol{\varphi}(t)=$ $\left(\varphi\left(t_{i}\right)\right)_{1 \leq i \leq n}$.

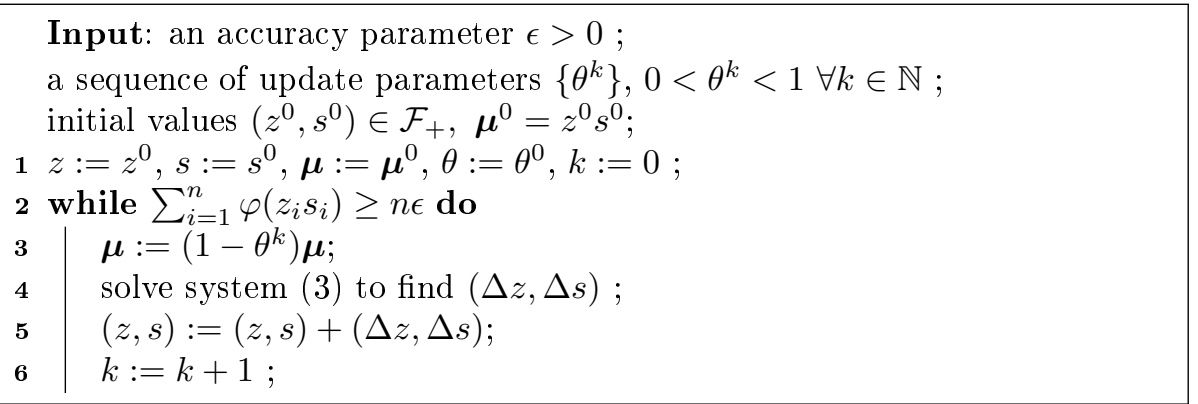

Algorithm 2: $\varphi$-Full Newton step IPM ( $\varphi$-FN-IPM)

One important characteristic of $\varphi$ is the constant $T$ defined by

$$
-\varphi^{\prime \prime}(0)=T\left(\varphi^{\prime}(0)\right)^{2} .
$$

By conditions (iv) and (v), $T \geq 0$ and $T=0$ for $\varphi(t)=t$. It is to be noted that arbitrary values of $T>0$ can be achieved by scaling $\varphi$.

Note that $t \mapsto \sqrt{t}$, in the same way as any function $t \mapsto t^{q}, 0<q<1$, does not satisfy these hypotheses since it is not differentiable in 0 . However we can consider a smooth version for $\epsilon>0$ with $t \mapsto(t+\epsilon)^{q}-(\epsilon)^{q}$. As said in the introduction the classical method is given by $\varphi(t)=t$. Other examples are $\varphi: t \mapsto \log (1+t)$ and functions constructed as in [15, ?]: for instance $\varphi: t \mapsto \frac{t}{t+1}$ and $\varphi: t \mapsto 1-\exp (-t)$. It is interesting to note that in the two latter cases, the function $\varphi$ is bounded. Moreover, notice that if a function $\varphi$ satisfies all these hypotheses, then $t \mapsto \alpha \varphi(C t)$ with $\alpha, C \in \mathbb{R}_{++}$also satisfies these hypotheses.

The main result of this section (Theorem 3.5 states the polynomial worstcase complexity of the $\varphi$-FN-IPM described in Algorithm 2. In order to get to this result we define a measure of the proximity to the central path in Section 3.1 . Then, in Section 3.2. Theorem 3.1 estimates the error made at each Newton step. Sections 3.3 and 3.4 present conditions to ensure the correct behaviour of the algorithm: strict feasibility of the iterates (Theorem 3.2) and quadratic convergence of the Newton process (Theorem 3.3). Section 3.5 provides the sequence of update parameters (Theorem 3.4). 


\subsection{Proximity measure}

At each iteration, after updating parameter $\boldsymbol{\mu}$, we compute the Newton direction $(\Delta z, \Delta s)$ as a solution of system (3). Then we update the iterates with

$$
z^{+}=z+\alpha \Delta z \text { and } s^{+}=s+\alpha \Delta s .
$$

Note that here we consider a damping factor $\alpha \in[0,1]$ to be more general. In this case we denote $(\alpha \Delta z, \alpha \Delta s)$ the $\alpha$-Newton step and the full Newton step is given for $\alpha=1$.

In order to measure the distance to the target on the central path we consider a proximity measure $\delta_{\varphi}(z, s, \boldsymbol{\mu})$ defined by

$$
\delta_{\varphi}(z, s, \boldsymbol{\mu}):=\frac{1}{2} \| \frac{\varphi^{\prime}(0)}{\boldsymbol{\varphi}^{\prime}(z s)}\left(\left(v_{\varphi}(z, s, \boldsymbol{\mu})^{-1}-v_{\varphi}(z, s, \boldsymbol{\mu})\right) \|_{2},\right.
$$

with

$$
v_{\varphi}(z, s, \boldsymbol{\mu}):=\sqrt{\frac{\varphi(z s)}{\boldsymbol{\mu}}} \text { and } v_{\varphi}(z, s, \boldsymbol{\mu})^{-1}:=\sqrt{\frac{\boldsymbol{\mu}}{\varphi(z s)}} .
$$

We may omit the arguments of $v_{\varphi}(z, s, \boldsymbol{\mu})$ and $\delta_{\varphi}(z, s, \boldsymbol{\mu})$, when it is clear from the context. Notice that this proximity measure is a generalization of the one in [27] since they consider

$$
\delta_{t \mapsto t}:=\frac{1}{2}\left\|\sqrt{\frac{\boldsymbol{\mu}}{z s}}-\sqrt{\frac{z s}{\boldsymbol{\mu}}}\right\|_{2}
$$

Both proximity measures are equal for $\varphi(t)=t$. Moreover for any function $\varphi$, the two proximity measures are asymptotically similar (for $z s \downarrow 0$ ).

The following two lemmas link the iterates and the proximity measure.

Lemma 3.1. If $(z, s) \in \mathcal{F}_{+}$and $\delta_{\varphi} \leq 1$, then $\varphi(z s) \leq 6 \mu$.

Proof. Assume by contradiction that there exists $i \in\{1, \ldots, n\}$ such that $\varphi\left(z_{i} s_{i}\right)>6 \boldsymbol{\mu}_{i}$. Since $\varphi$ is increasing and $z s>0$ :

$$
\begin{aligned}
2 \delta_{\varphi} \geq\left\|v_{\varphi}{ }^{-1}-v_{\varphi}\right\|_{2}=\left\|\frac{\sqrt{\boldsymbol{\mu}}}{\sqrt{\varphi(z s)}}-\frac{\sqrt{\varphi(z s)}}{\sqrt{\boldsymbol{\mu}}}\right\|_{2} & \geq\left|\frac{\sqrt{\boldsymbol{\mu}_{i}}}{\sqrt{\varphi\left(z_{i} s_{i}\right)}}-\frac{\sqrt{\varphi\left(z_{i} s_{i}\right)}}{\sqrt{\boldsymbol{\mu}_{i}}}\right| \\
& =\frac{\sqrt{\varphi\left(z_{i} s_{i}\right)}}{\sqrt{\boldsymbol{\mu}_{i}}}-\frac{\sqrt{\boldsymbol{\mu}_{i}}}{\sqrt{\varphi\left(z_{i} s_{i}\right)}} \\
& >\sqrt{6}-\frac{1}{\sqrt{6}} \approx 2,04,
\end{aligned}
$$

where the penultimate step comes from the increasing property of the function $x \mapsto x-1 / x$ on $\mathbb{R}_{++}$. This is in contradiction with $\delta_{\varphi} \leq 1$.

The following lemma gives bounds on $\delta_{\varphi}$. 
Lemma 3.2. Let $\delta=\frac{1}{2}\left\|v_{\varphi}{ }^{-1}-v_{\varphi}\right\|_{2}$, then

$$
\delta \leq \delta_{\varphi} \leq\left(1-\frac{\varphi^{\prime \prime}(0)\|z s\|_{\infty}}{\varphi^{\prime}\left(\|z s\|_{\infty}\right)}\right) \delta .
$$

Furthermore in a close neighbourhood of the central path, i.e $\delta_{\varphi} \leq 1$, we have

$$
\delta \leq \delta_{\varphi} \leq\left(1-\frac{\varphi^{\prime \prime}(0) \varphi^{-1}\left(6\|\boldsymbol{\mu}\|_{\infty}\right)}{\varphi^{\prime}\left(\varphi^{-1}\left(6\|\boldsymbol{\mu}\|_{\infty}\right)\right)}\right) \delta:=\Gamma\left(\|\boldsymbol{\mu}\|_{\infty}\right) \delta .
$$

Proof. By convexity of function $\varphi^{\prime}$ for all $i \in\{1, \ldots, n\}$

$$
\varphi^{\prime}\left(z_{i} s_{i}\right) \geq \varphi^{\prime}(0)+\varphi^{\prime \prime}(0) z_{i} s_{i} .
$$

Then, for all $i \in\{1, \ldots, n\}$

$$
1 \leq \frac{\varphi^{\prime}(0)}{\varphi^{\prime}\left(z_{i} s_{i}\right)} \leq 1-\frac{\varphi^{\prime \prime}(0) z_{i} s_{i}}{\varphi^{\prime}\left(z_{i} s_{i}\right)} \leq \max _{i}\left(1-\frac{\varphi^{\prime \prime}(0) z_{i} s_{i}}{\varphi^{\prime}\left(z_{i} s_{i}\right)}\right) .
$$

Hence, by definition of $\delta$ and $\delta_{\varphi}$

$$
\delta \leq \delta_{\varphi} \leq\left(1-\frac{\varphi^{\prime \prime}(0)\|z s\|_{\infty}}{\varphi^{\prime}\left(\|z s\|_{\infty}\right)}\right) \delta .
$$

The sharpest result in a close neighbourhood of the central path is deduced from Lemma 3.1 .

In the previous lemma we define $\Gamma$ as a function of $\|\boldsymbol{\mu}\|_{\infty}$ which depends on the choice of $\varphi$ as

$$
\Gamma\left(\|\boldsymbol{\mu}\|_{\infty}\right)=\left(1-\frac{\varphi^{\prime \prime}(0) \varphi^{-1}\left(6\|\boldsymbol{\mu}\|_{\infty}\right)}{\varphi^{\prime}\left(\varphi^{-1}\left(6\|\boldsymbol{\mu}\|_{\infty}\right)\right)}\right) .
$$

It is easily seen that for $\varphi(t)=t$ then $\Gamma\left(\|\boldsymbol{\mu}\|_{\infty}\right)=1$ for all $\|\boldsymbol{\mu}\|_{\infty}$. Moreover, for any function $\varphi, \Gamma$ is increasing with respect to $\|\boldsymbol{\mu}\|_{\infty}$, and converges to 1 as $\|\boldsymbol{\mu}\|_{\infty} \downarrow 0$.

\subsection{Error bound of the Newton step}

We use the first order Taylor-Lagrange formula applied to $\varphi$ in $z s$. There exists $\xi \in\left[z^{+} s^{+}, z s\right]$ such that

$$
\begin{aligned}
\varphi\left(z^{+} s^{+}\right) & =\varphi(z s)+\alpha \varphi^{\prime}(z s)(z \Delta s+s \Delta z) \\
& +\alpha^{2} \Delta z \Delta s \varphi^{\prime}(z s)+\frac{\varphi^{\prime \prime}(\xi)}{2}\left(z^{+} s^{+}-z s\right)^{2},
\end{aligned}
$$

with $(\Delta z, \Delta s)$ solution of (3). The update of $\boldsymbol{\mu}$ is chosen such that $z^{+} s^{+}<z s$. Therefore the error we make when we say that $\varphi\left(z^{+} s^{+}\right)$is the $\mu$-center is

$$
\eta:=\alpha^{2} \Delta z \Delta s \varphi^{\prime}(z s)+\frac{\varphi^{\prime \prime}(\xi)}{2}\left(z^{+} s^{+}-z s\right)^{2} .
$$

The following sequence of lemmas aims to bound this error in terms of the proximity measure. We first state a lemma from [16]. 
Lemma 3.3 (Lemma 5.1, [16]). Let $(z, s) \in \mathcal{F}_{+}$and $a \in \mathbb{R}^{n}$. Assume that matrix $M$ is a positive semidefinite matrix. Let $(\Delta z, \Delta s)$ be the solution of

$$
\left\{\begin{array}{l}
-M \Delta z+\Delta s=0 \\
s \Delta z+z \Delta s=a
\end{array} .\right.
$$

Then

$$
\begin{gathered}
\|\Delta z \Delta s\|_{2} \leq C_{2}\left\|\frac{a}{\sqrt{z s}}\right\|_{2}^{2}, \\
\|\Delta z \Delta s\|_{\infty} \leq C_{\infty}\left\|\frac{a}{\sqrt{z s}}\right\|_{2}^{2},
\end{gathered}
$$

with $C_{2}=1 /(2 \sqrt{2})$ and $C_{\infty}=1 / 4$.

Straightforward application of this lemma for $\mathrm{a}=\frac{\mu-\varphi(z s)}{\varphi^{\prime}(z s)}$ and $v_{\varphi}=\sqrt{\frac{\varphi(z s)}{\mu}}$ gives the following lemma.

Lemma 3.4. Let $(z, s) \in \mathcal{F}_{+},(\Delta z, \Delta s)$ solution of $(3)$ and $p \in\{2, \infty\}$, then

$$
\|\Delta z \Delta s\|_{p} \leq C_{p}\left\|\frac{\sqrt{\varphi(z s)} \sqrt{\boldsymbol{\mu}}}{\sqrt{z s} \varphi^{\prime}(z s)}\left(v_{\varphi}{ }^{-1}-v_{\varphi}\right)\right\|_{2}^{2} .
$$

The next lemma will bound $\left\|\Delta z \Delta s \varphi^{\prime}(z s)\right\|_{p}$ for $p \in\{2, \infty\}$.

Lemma 3.5. Let $(z, s) \in \mathcal{F}_{+},(\Delta z, \Delta s)$ solution of $(3)$ and $p \in\{2, \infty\}$, then

$$
\left\|\Delta z \Delta s \varphi^{\prime}(z s)\right\|_{p} \leq 4 C_{p}\|\boldsymbol{\mu}\|_{\infty} \delta_{\varphi}^{2} .
$$

Proof. By concavity of $\varphi$ we have that $\varphi\left(z_{i} s_{i}\right) \leq \varphi^{\prime}(0) z_{i} s_{i}, \forall i$, so

$$
\frac{\varphi(z s) \varphi^{\prime}(0)}{z s\left(\varphi^{\prime}(z s)\right)^{2}} \leq \frac{\left(\varphi^{\prime}(0)\right)^{2}}{\left(\varphi^{\prime}(z s)\right)^{2}} .
$$

Furthermore for $p \in\{2, \infty\}$ and using Lemma 3.4 followed by (8)

$$
\begin{aligned}
\left\|\Delta z \Delta s \varphi^{\prime}(z s)\right\|_{p} & \leq\|\Delta z \Delta s\|_{p} \boldsymbol{\varphi}^{\prime}(0) \\
& \leq C_{p}\left\|\frac{\sqrt{\boldsymbol{\varphi}(z s)} \sqrt{\boldsymbol{\mu}}}{\sqrt{z s} \boldsymbol{\varphi}^{\prime}(z s)}\left(v^{-1}-v\right)\right\|_{2}^{2} \boldsymbol{\varphi}^{\prime}(0) \\
& \leq\left(\sum_{i=1}^{n} C_{p} \frac{\boldsymbol{\varphi}\left(z_{i} s_{i}\right) \boldsymbol{\mu}_{i}}{z_{i} s_{i}\left(\boldsymbol{\varphi}^{\prime}\left(z_{i} s_{i}\right)\right)^{2}}\left(v_{\varphi_{i}}^{-1}-v_{\varphi_{i}}\right)^{2}\right) \boldsymbol{\varphi}^{\prime}(0) \\
& \leq \sum_{i=1}^{n} C_{p} \boldsymbol{\mu}_{i}\left(\frac{\boldsymbol{\varphi}^{\prime}(0)}{\boldsymbol{\varphi}^{\prime}\left(z_{i} s_{i}\right)}\right)^{2}\left(v_{\varphi_{i}}{ }^{-1}-v_{\varphi_{i}}\right)^{2} \\
& \leq 4 C_{p}\|\boldsymbol{\mu}\|_{\infty} \delta_{\varphi}^{2}
\end{aligned}
$$


Now we move to the main result which gives a bound for the complete error.

Lemma 3.6. Let $(z, s) \in \mathcal{F}_{+}$and $(\Delta z, \Delta s)$ solution of $(3)$. For $p \in\{2, \infty\}$ we have

$\|\eta\|_{p} \leq\left(4 C_{p}+2 T \varphi\left(\|z s\|_{\infty}\right)\right) \alpha^{2}\|\boldsymbol{\mu}\|_{\infty} \delta_{\varphi}^{2}+\left(C_{\infty} \sqrt{\varphi\left(\|z s\|_{\infty}\right)}+\alpha C_{p}^{2} \sqrt{\|\boldsymbol{\mu}\|_{\infty}} \delta_{\varphi}\right) 8 \alpha^{3} T\|\boldsymbol{\mu}\|_{\infty}{ }^{3 / 2} \delta_{\varphi}^{3}$

Proof. By equation (7) we have

$$
\begin{aligned}
\eta= & \alpha^{2} \Delta z \Delta s \varphi^{\prime}(z s)+\frac{\alpha^{2} \varphi^{\prime \prime}(\xi)}{2}(z \Delta s+s \Delta z)^{2}+\frac{\alpha^{4} \varphi^{\prime \prime}(\xi)}{2}(\Delta s \Delta z)^{2} \\
& +\alpha^{3} \varphi^{\prime \prime}(\xi)(\Delta s \Delta z)(z \Delta s+s \Delta z)
\end{aligned}
$$

By considering the norm for $p \in\{2, \infty\}$ and triangle inequalities

$$
\begin{aligned}
\|\eta\|_{p} \leq & \alpha^{2}\left\|\Delta z \Delta s \varphi^{\prime}(z s)\right\|_{p}+\alpha^{2}\left\|\frac{\varphi^{\prime \prime}(\xi)}{2}(z \Delta s+s \Delta z)^{2}\right\|_{p}+\alpha^{4}\left\|\frac{\varphi^{\prime \prime}(\xi)}{2}(\Delta s \Delta z)^{2}\right\|_{p} . \\
& +\alpha^{3}\left\|\varphi^{\prime \prime}(\xi)(\Delta s \Delta z)(z \Delta s+s \Delta z)\right\|_{p}
\end{aligned}
$$

We now bound each term of the above right-hand side. First, Lemma 3.5 gives

$$
\left\|\Delta z \Delta s \varphi^{\prime}(z s)\right\|_{p} \leq 4 C_{p}\|\boldsymbol{\mu}\|_{\infty} \delta_{\varphi}^{2} .
$$

Using successively $-\boldsymbol{\varphi}^{\prime \prime}(0)=T\left(\boldsymbol{\varphi}^{\prime}(0)\right)^{2},(\boldsymbol{\mu}-\boldsymbol{\varphi}(z s))^{2}=\left(\boldsymbol{\mu} v_{\varphi}\left(v_{\varphi}{ }^{-1}-v_{\varphi}\right)\right)^{2} \leq$ $\boldsymbol{\mu} \varphi(z s)\left(v_{\varphi}{ }^{-1}-v_{\varphi}\right)^{2}$ and $\varphi\left(z_{i} s_{i}\right) \leq \varphi\left(\|z s\|_{\infty}\right), \forall i \in\{1, \ldots, n\}$, we obtain step by step

$$
\begin{aligned}
\left\|\frac{\varphi^{\prime \prime}(\xi)}{2}(z \Delta s+s \Delta z)^{2}\right\|_{p} & \leq-\frac{\varphi^{\prime \prime}(0)}{2}\left\|(z \Delta s+s \Delta z)^{2}\right\|_{p} \\
& =\frac{T}{2}\left\|\left(\frac{\varphi^{\prime}(0)}{\boldsymbol{\varphi}^{\prime}(z s)}\right)^{2}(\boldsymbol{\mu}-\boldsymbol{\varphi}(z s))^{2}\right\|_{p} \\
& \leq \frac{T \varphi\left(\|z s\|_{\infty}\right)\|\boldsymbol{\mu}\|_{\infty}}{2}\left\|\left(\frac{\varphi^{\prime}(0)}{\boldsymbol{\varphi}^{\prime}(z s)}\left(v_{\varphi}^{-1}-v_{\varphi}\right)\right)^{2}\right\|_{p} \\
& \leq 2 T \varphi\left(\|z s\|_{\infty}\right)\|\boldsymbol{\mu}\|_{\infty} \delta_{\varphi}^{2} .
\end{aligned}
$$

For the third term, we use Lemma 3.5 and the following two properties: $-\varphi^{\prime \prime}(0)=$ $T\left(\varphi^{\prime}(0)\right)^{2}$ and $\left\|u^{2}\right\|_{p} \leq\|u\|_{p}^{2} \forall u \in \mathbb{R}^{n}$

$$
\begin{aligned}
\left\|\frac{\varphi^{\prime \prime}(\xi)}{2}(\Delta s \Delta z)^{2}\right\|_{p} & \leq \frac{T}{2}\left\|\left(\boldsymbol{\varphi}^{\prime}(0) \Delta s \Delta z\right)^{2}\right\|_{p} \\
& \leq \frac{T}{2}\left\|\varphi^{\prime}(0) \Delta s \Delta z\right\|_{p}^{2} \\
& \leq \frac{T}{2}\left(\|\boldsymbol{\mu}\|_{\infty} 4 C_{p} \delta_{\varphi}^{2}\right)^{2}=8 T C_{p}^{2}\|\boldsymbol{\mu}\|_{\infty}^{2} \delta_{\varphi}^{4} .
\end{aligned}
$$


Finally, the definition of $v_{\varphi}$ implies that

$$
\begin{aligned}
\left\|\varphi^{\prime}(0)(z \Delta s+s \Delta z)\right\|_{p} & =\left\|\frac{\varphi^{\prime}(0)}{\varphi^{\prime}(z s)} \boldsymbol{\mu} v_{\varphi}\left(v_{\varphi}^{-1}-v_{\varphi}\right)\right\|_{p} \\
& \leq\left\|\frac{\varphi^{\prime}(0)}{\varphi^{\prime}(z s)} \sqrt{\|\boldsymbol{\mu}\|_{\infty}} \sqrt{\varphi\left(\|z s\|_{\infty}\right)}\left(v_{\varphi}^{-1}-v_{\varphi}\right)\right\|_{p},
\end{aligned}
$$

Using the above inequality, as well as $\|u w\|_{p} \leq\|u\|_{\infty}\|w\|_{p}, \forall(u, w) \in \mathbb{R}^{2 n}$ and $-\varphi^{\prime \prime}(0)=T\left(\varphi^{\prime}(0)\right)^{2}$, we get

$$
\begin{aligned}
\left\|\varphi^{\prime \prime}(\xi)(\Delta s \Delta z)(z \Delta s+s \Delta z)\right\|_{p} & \leq T\left\|\varphi^{\prime}(0)(\Delta s \Delta z)\right\|_{\infty}\left\|\varphi^{\prime}(0)(z \Delta s+s \Delta z)\right\|_{p} \\
& \leq 8 T C_{\infty} \sqrt{\varphi\left(\|z s\|_{\infty}\right)}\|\boldsymbol{\mu}\|_{\infty}{ }^{3 / 2} \delta_{\varphi}^{3}
\end{aligned}
$$

which completes the proof.

In the special case where we are in a close neighbourhood of the central path we get an improved version of the result:

Theorem 3.1. Let $(z, s) \in \mathcal{F}_{+}$and $\delta_{\varphi} \leq 1$. Given $(\Delta z, \Delta s)$ solutions of (3) and $p \in\{2, \infty\}$, we have

$$
\|\eta\|_{p} \leq\left(4 C_{p}+12 T\|\boldsymbol{\mu}\|_{\infty}\right) \alpha^{2}\|\boldsymbol{\mu}\|_{\infty} \delta_{\varphi}^{2}+\left(\sqrt{6} C_{\infty}+\alpha C_{p}^{2} \delta_{\varphi}\right) \alpha^{3} 8 T\|\boldsymbol{\mu}\|_{\infty}{ }^{2} \delta_{\varphi}^{3}
$$

Proof. The proof is the same as that of Lemma 3.6. but instead of using $\varphi\left(z_{i} s_{i}\right) \leq \varphi\left(\|z s\|_{\infty}\right), \forall i$ we can use $\boldsymbol{\varphi}(z s) \leq 6 \boldsymbol{\mu}$ from Lemma 3.1 .

For instance, for $\alpha=1$, using that $\delta_{\varphi}^{p} \leq \delta_{\varphi}$ for $p \geq 1$, then $(9)$ becomes

$$
\|\eta\|_{\infty} \leq\left(\|\boldsymbol{\mu}\|_{\infty}+\left(\frac{25+4 \sqrt{6}}{2}\right) T\|\boldsymbol{\mu}\|_{\infty}^{2}\right) \delta_{\varphi}^{2} .
$$

For $\varphi(t)=t$ we get the same result as in 25

$$
\|\eta\|_{\infty} \leq\|\boldsymbol{\mu}\|_{\infty} \delta_{\varphi}^{2} .
$$

\subsection{Feasibility of the Newton step}

A Newton step is feasible (strictly feasible) if the couple $\left(z^{+}, s^{+}\right)$defined by (4) is feasible (strictly feasible).

Theorem 3.2. Let $\alpha$ be in [0,1] and $\delta_{\varphi} \leq 1$. The $\alpha$-Newton step is strictly feasible for $(z, s)$ strictly feasible if

$$
\alpha \delta_{\varphi}^{2}<\frac{1}{1+\left(\frac{25+4 \sqrt{6}}{2}\right) T\|\boldsymbol{\mu}\|_{\infty}}
$$

Furthermore if this condition holds for $\alpha=1$, then the full Newton step is strictly feasible. 
For $\alpha=1$ condition (10) implies that the proximity measure, $\delta_{\varphi}$, must be less than 1 , which will not be a restrictive assumption.

Proof. For $\alpha=0$ the result trivially holds. Therefore, we can assume without loss of generality that $\alpha \in] 0,1]$. Let $\beta \in] 0, \alpha]$, and

$$
z^{\beta}=z+\beta \Delta z, s^{\beta}=s+\beta \Delta s,
$$

where $(\Delta z, \Delta s)$ is the solution of $(3)$. The proof rests upon a continuity argument. Indeed, we assume $z, s>0$ so $\varphi(z s)>0$ and, using equations (3) and (6):

$$
\begin{aligned}
\boldsymbol{\varphi}\left(z^{\beta} s^{\beta}\right) & =\boldsymbol{\varphi}(z s)+\beta \boldsymbol{\varphi}^{\prime}(z s)(z \Delta s+s \Delta z)+\eta(\beta), \\
& =\boldsymbol{\varphi}(z s)(1-\beta)+\beta\left(\boldsymbol{\mu}+\frac{\eta(\beta)}{\beta}\right), \\
& \geq \boldsymbol{\varphi}(z s)(1-\beta)+\beta\left(\boldsymbol{\mu}-\boldsymbol{e} \frac{\|\eta(\beta)\|_{\infty}}{\beta}\right),
\end{aligned}
$$

which is positive for all $z, s>0$ if

$$
\|\boldsymbol{\mu}\|_{\infty}>\frac{\|\eta(\beta)\|_{\infty}}{\beta}
$$

Lemma 3.6 provides that this condition holds if

$$
\|\boldsymbol{\mu}\|_{\infty}>\left(4 C_{\infty}+2 T \varphi\left(\|z s\|_{\infty}\right)\right) \beta\|\boldsymbol{\mu}\|_{\infty} \delta_{\varphi}^{2}+\left(C_{\infty} \sqrt{\varphi\left(\|z s\|_{\infty}\right)}+\beta C_{\infty}^{2} \sqrt{\|\boldsymbol{\mu}\|_{\infty}} \delta_{\varphi}\right) 8 \beta^{2} T\|\boldsymbol{\mu}\|_{\infty}{ }^{3 / 2} \delta_{\varphi}^{3} .
$$

The right-hand side is increasing with respect to $\beta$, so it is sufficient to verify

$$
\|\boldsymbol{\mu}\|_{\infty}>\left(4 C_{\infty}+2 T \varphi\left(\|z s\|_{\infty}\right)\right) \alpha\|\boldsymbol{\mu}\|_{\infty} \delta_{\varphi}^{2}+\left(C_{\infty} \sqrt{\varphi\left(\|z s\|_{\infty}\right)}+\alpha C_{\infty}^{2} \sqrt{\|\boldsymbol{\mu}\|_{\infty}} \delta_{\varphi}\right) 8 \alpha^{2} T\|\boldsymbol{\mu}\|_{\infty}{ }^{3 / 2} \delta_{\varphi}^{3} .
$$

Therefore, since $\delta_{\varphi} \leq 1$ and according to Theorem 3.1 strict feasibility is satisfied if

$$
1-\delta_{\varphi}^{2} \alpha\left(4 C_{\infty}+\left(12+8 \sqrt{6} C_{\infty}+8 C_{\infty}^{2}\right) T\|\boldsymbol{\mu}\|_{\infty}\right)>0,
$$

which corresponds to one of our assumptions. It follows that for all $\beta \in[0, \alpha]$, $\varphi\left(z^{\beta} s^{\beta}\right)>0$. By continuity of $\varphi$, this implies that none of $z^{\beta}$ or $s^{\beta}$ vanish for $\beta \in[0, \alpha]$, so the result follows.

Figure 2 illustrates Theorem 3.2 with two examples of update after a Newton step. It appears that starting from a point with a proximity measure greater than one step can lead outside the feasible domain.

\subsection{Quadratic decrease of the proximity measure}

The Newton method is known to behave well in a close neighbourhood of the solution. The following theorem states a condition on the proximity measure, that ensures a quadratic convergence of the Newton step. 


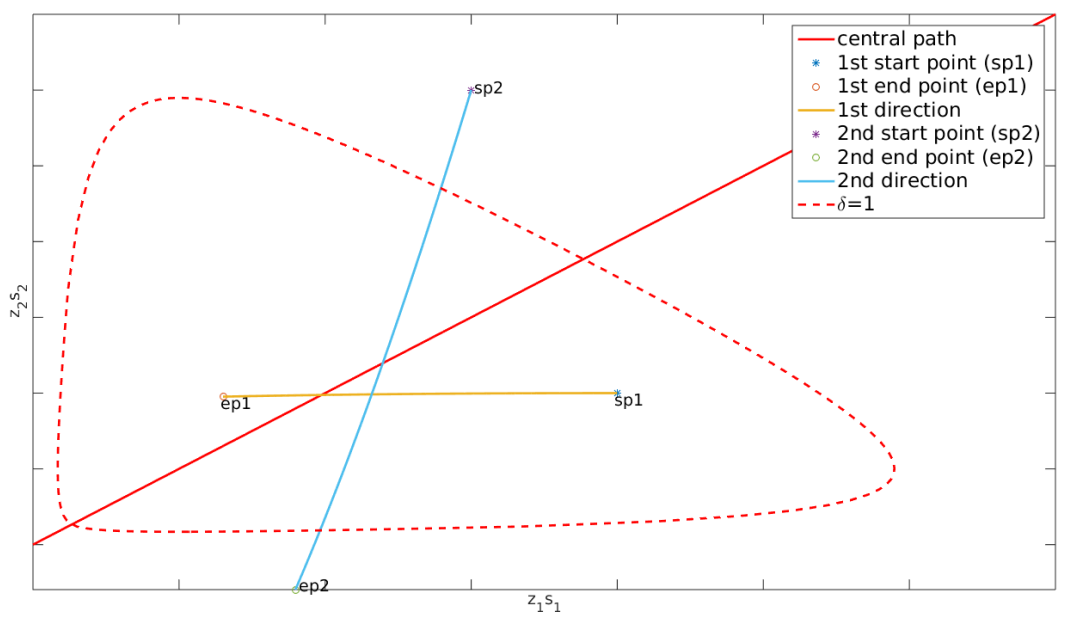

Figure 2: Illustration of Theorem 3.2

Theorem 3.3. Assume $(z, s) \in \mathcal{F}_{+}$and $\left(z^{+}, s^{+}\right)$be defined as in (4) for $\alpha=1$. Let $\delta_{\varphi}:=\delta_{\varphi}(z, s, \boldsymbol{\mu}), \delta_{\varphi}^{+}:=\delta_{\varphi}\left(z^{+}, s^{+}, \boldsymbol{\mu}\right), \Gamma\left(\|\boldsymbol{\mu}\|_{\infty}\right)$ defined in (5) and

$Q\left(\|\boldsymbol{\mu}\|_{\infty}\right):=\frac{1}{1+\left(\frac{25+4 \sqrt{6}}{2}\right) T\|\boldsymbol{\mu}\|_{\infty}}\left(1-\frac{\left(\Gamma\left(\|\boldsymbol{\mu}\|_{\infty}\right)\left(\sqrt{2}+(13+2 \sqrt{6}) T\|\boldsymbol{\mu}\|_{\infty}\right)\right)^{2}}{4}\right)$

If

$$
\delta_{\varphi}^{2} \leq Q\left(\|\boldsymbol{\mu}\|_{\infty}\right)
$$

then

$$
\delta_{\varphi}^{+} \leq \delta_{\varphi}^{2}
$$

Proof. Let $\Gamma=\Gamma\left(\|\boldsymbol{\mu}\|_{\infty}\right)$ be the constant defined in Lemma 3.2 .

$$
\begin{aligned}
\delta_{\varphi}^{+} \leq \frac{\Gamma}{2}\left\|\left(v_{\varphi}{ }^{+}\right)^{-1}-\left(v_{\varphi}^{+}\right)\right\|_{2} & =\frac{\Gamma}{2}\left\|\left(v_{\varphi}^{+}\right)^{-1}\left(\mathbf{e}-\left(v_{\varphi}^{+}\right)^{2}\right)\right\|_{2} \\
& \leq \frac{\Gamma}{2}\left\|\frac{\frac{\eta}{\mu}}{\sqrt{\mathbf{e}+\frac{\eta}{\mu}}}\right\|_{2} \leq \frac{\Gamma\left\|\frac{\eta}{\mu}\right\|_{2}}{2 \sqrt{1-\left\|\frac{\eta}{\mu}\right\|_{\infty}}} .
\end{aligned}
$$

Now, using Theorem 3.1 with a full step, i.e $\alpha=1$, it follows

$$
\delta_{\varphi}^{+} \leq \delta_{\varphi}^{2} \Gamma \frac{4 C_{2}+\left(12+8 \sqrt{6} C_{\infty}+8 C_{2}^{2}\right) T\|\boldsymbol{\mu}\|_{\infty}}{2 \sqrt{1-\delta_{\varphi}^{2}\left(4 C_{\infty}+\left(12+8 \sqrt{6} C_{\infty}+8 C_{\infty}^{2}\right) T\|\boldsymbol{\mu}\|_{\infty}\right)}} .
$$

So, $\delta_{\varphi}^{+} \leq \delta_{\varphi}^{2}$ if $\delta_{\varphi}^{2} \leq Q\left(\|\boldsymbol{\mu}\|_{\infty}\right)$. 
[25]:

Considering $\varphi(t)=t$, the condition of Theorem 3.3 becomes the same as in

$$
\delta_{\varphi}^{2} \leq \frac{1}{2} .
$$

Figure 3 presents the regions $\delta_{\varphi} \leq \frac{1}{\sqrt{2}}, \delta_{\varphi} \leq \frac{1}{2}$ and illustrate one Newton step from a region to the other.

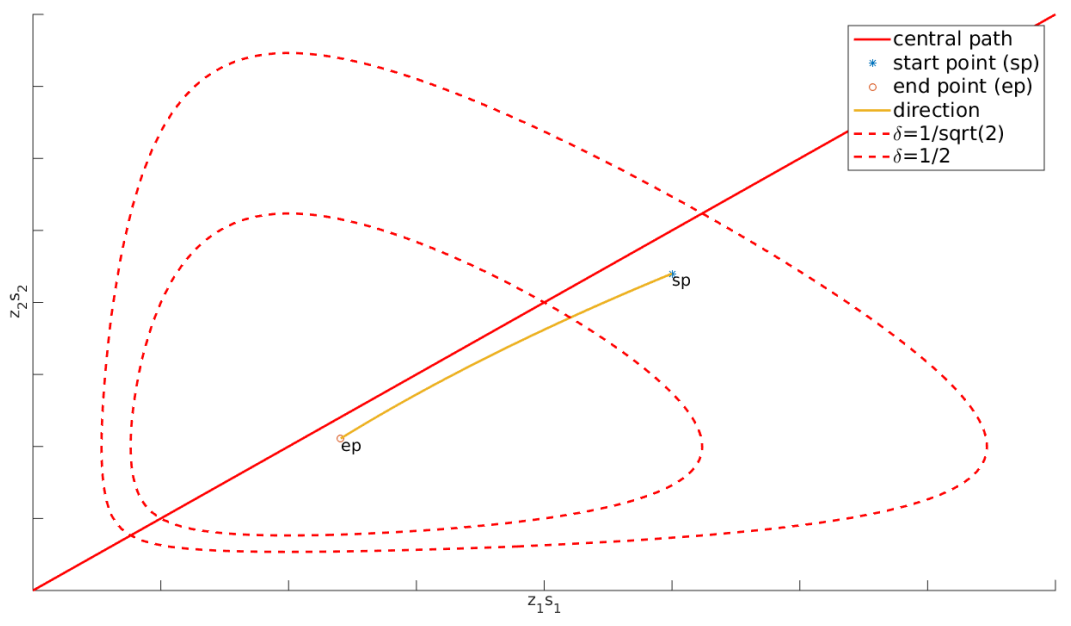

Figure 3: Illustration of Theorem 3.3

Remark 3.1. The condition in Theorem 3.3 implies the condition in Theorem 3.2 So, if the iterates locate in the neighbourhood of quadratic convergence, the full Newton step will provide strictly feasible iterates.

Remark 3.2. Notice that since the proximity measure is always non-negative, the condition from Theorem 3.3 can hold only when $\mu$ is sufficiently small, i.e. when

$$
\Gamma\left(\|\boldsymbol{\mu}\|_{\infty}\right)\left(\sqrt{2}+(13+2 \sqrt{6}) T\|\boldsymbol{\mu}\|_{\infty}\right) \leq 2 .
$$

This is not a restrictive assumption, since given an initial point we can use a scaling technique so that this point satisfy this condition. This technique will be clarified numerically in Section 4

\subsection{Updating parameter strategy}

The sequence of parameter $\left\{\theta^{k}\right\}$ must be chosen such that the iterates remain strictly feasible and satisfy the condition of Theorem 3.3 . In this section, Proposition 3.1 gives an upper bound on the proximity measure after an update on 
$\boldsymbol{\mu}$, that is $\boldsymbol{\mu}^{+}=\boldsymbol{\mu}\left(1-\theta^{k}\right)$, and then Theorem 3.4 describes how to build the sequence $\left\{\theta^{k}\right\}$.

First, we provide a useful lemma.

Lemma 3.7. Let $(z, s) \in \mathcal{F}_{+},(\Delta z, \Delta s)$ solution of (3) and $\delta_{\varphi} \leq 1$. Then,

$$
\varphi\left(z^{+} s^{+}\right) \leq 2 \mu .
$$

Proof. From equation (6) and concavity of function $\varphi$, it holds

$$
\varphi\left(z^{+} s^{+}\right) \leq \boldsymbol{\mu}+\Delta z \Delta s \varphi^{\prime}(z s) .
$$

So that by Lemma 3.5 , it follows

$$
\boldsymbol{\varphi}\left(z^{+} s^{+}\right) \leq \boldsymbol{\mu}\left(1+4 C_{\infty} \delta_{\varphi}^{2}\right) \leq 2 \boldsymbol{\mu} .
$$

Now, the following proposition provides an upper bound of the proximity measure after an update of the parameter, denoted $\delta_{\varphi}^{+}$, in terms of the update $\theta^{k}$ and the proximity measure before this update, denoted $\overline{\delta_{\varphi}}$.

Proposition 3.1. Let $\overline{v_{\varphi}}:=v_{\varphi}\left(z^{+}, s^{+}, \boldsymbol{\mu}\right)$ and $v_{\varphi}:=\left(z^{+}, s^{+}, \boldsymbol{\mu}^{+}\right)$, where $\boldsymbol{\mu}^{+}:=$ $\left(1-\theta^{k}\right) \boldsymbol{\mu}$. Then,

$$
\left(\delta_{\varphi}^{+}\right)^{2} \leq\left(1-\theta^{k}\right)\left(\overline{\delta_{\varphi}}\right)^{2}+\frac{\Gamma\left(\|\boldsymbol{\mu}\|_{\infty}\right)^{2} n\left(\theta^{k}\right)^{2}}{2\left(1-\theta^{k}\right)} .
$$

Proof. Noticing that

$$
v_{\varphi}=\frac{\bar{\varphi}_{\varphi}}{\sqrt{1-\theta^{k}}}
$$

it follows that

$$
\begin{aligned}
\left(\delta_{\varphi}^{+}\right)^{2}= & \frac{1}{4} \sum_{i=1}^{n}\left(\frac{\varphi^{\prime}(0)}{\boldsymbol{\varphi}_{i}^{\prime}(z s)}\right)^{2}\left(v_{\varphi_{i}}^{-2}+v_{\varphi_{i}}^{2}-2\right) \\
= & \frac{1}{4} \sum_{i=1}^{n}\left(\frac{\varphi^{\prime}(0)}{\boldsymbol{\varphi}_{i}^{\prime}(z s)}\right)^{2}\left((1-\theta){\overline{v_{\varphi}}}^{-2}+\frac{{\overline{\varphi_{\varphi}}}_{i}^{2}}{(1-\theta)}-2\right) \\
= & (1-\theta)\left(\frac{1}{4} \sum_{i=1}^{n}\left(\frac{\varphi^{\prime}(0)}{\boldsymbol{\varphi}_{i}^{\prime}(z s)}\right)^{2}\left({\overline{\varphi_{\varphi}}}_{i}^{-2}+{\overline{v_{\varphi}}}_{i}^{2}-2\right)\right) \\
& +\frac{1}{4} \sum_{i=1}^{n}\left(\frac{\varphi^{\prime}(0)}{\boldsymbol{\varphi}_{i}^{\prime}(z s)}\right)^{2}\left(-2 \theta+\frac{2 \theta^{k}-\left(\theta^{k}\right)^{2}}{1-\theta^{k}} \bar{v}_{i}^{2}\right) \\
= & \left(1-\theta^{k}\right)\left(\overline{\delta_{\varphi}}\right)^{2}+\frac{1}{4} \sum_{i=1}^{n}\left(\frac{\varphi^{\prime}(0)}{\boldsymbol{\varphi}_{i}^{\prime}(z s)}\right)^{2}\left(-2 \theta^{k}+\frac{2 \theta^{k}-\left(\theta^{k}\right)^{2}}{1-\theta^{k}}{\overline{v_{\varphi}}}_{i}^{2}\right) \\
\leq & \left(1-\theta^{k}\right)\left(\overline{\delta_{\varphi}}\right)^{2}+\frac{\left(1+4 C_{\infty}\right) \Gamma\left(\|\boldsymbol{\mu}\|_{\infty}\right)^{2} n\left(\theta^{k}\right)^{2}}{4\left(1-\theta^{k}\right)}
\end{aligned}
$$

as $\Gamma\left(\|\boldsymbol{\mu}\|_{\infty}{ }^{+}\right) \leq \Gamma\left(\|\boldsymbol{\mu}\|_{\infty}\right)$ and by Lemma 3.7. 
We conclude this section, by a theorem, which describes the way the update parameters $\theta^{k}$ are chosen.

Theorem 3.4. Let $\boldsymbol{\mu}$ be such that $Q\left(\|\boldsymbol{\mu}\|_{\infty}\right)>0$ and $(z, s) \in \mathcal{F}_{+}$. Define $0<\theta^{k}<1$ as

$\theta^{k}=\frac{2 \sqrt{2 n \Gamma\left(\|\boldsymbol{\mu}\|_{\infty}\right)^{2}\left(-Q\left(\|\boldsymbol{\mu}\|_{\infty}\right)^{2}+Q\left(\|\boldsymbol{\mu}\|_{\infty}\right)\right)+Q\left(\|\boldsymbol{\mu}\|_{\infty}\right)^{2}}+2 Q\left(\|\boldsymbol{\mu}\|_{\infty}\right)^{2}-Q\left(\|\boldsymbol{\mu}\|_{\infty}\right)}{2 n \Gamma\left(\|\boldsymbol{\mu}\|_{\infty}\right)^{2}+4 Q\left(\|\boldsymbol{\mu}\|_{\infty}\right)^{2}}$.

The proximity measure $\delta_{\varphi}^{+}:=\delta_{\varphi}\left(z^{+}, s^{+}, \boldsymbol{\mu}\left(1-\theta^{k}\right)\right)$ satisfies the conditions of feasibility in Theorem 3.2 and quadratic convergence of the Newton step in Theorem 3.3 .

Proof. As pointed out earlier in Remark 3.1 the condition of Theorem 3.2 is weaker than the condition of Theorem 3.3 . Thus, it is sufficient to satisfy the former condition to ensure strict feasibility of the iterates.

According to the condition of Theorem 3.3 , after an update of $\boldsymbol{\mu}$, i.e $\boldsymbol{\mu}^{+}=$ $\left(1-\theta^{k}\right) \boldsymbol{\mu}$, the proximity measure $\delta_{\varphi}^{+}$must satisfy

$$
\left(\delta_{\varphi}^{+}\right)^{2} \leq Q\left(\|\boldsymbol{\mu}\|_{\infty}^{+}\right) .
$$

As $Q$ is decreasing with respect to $\|\boldsymbol{\mu}\|_{\infty}$, it is sufficient to ensure that

$$
\left(\delta_{\varphi}^{+}\right)^{2} \leq Q\left(\|\boldsymbol{\mu}\|_{\infty}\right) .
$$

By Proposition 3.1, we can choose any $\theta^{k}$ satisfying

$$
\left(1-\theta^{k}\right) Q\left(\|\boldsymbol{\mu}\|_{\infty}\right)^{2}+\frac{n \Gamma\left(\|\boldsymbol{\mu}\|_{\infty}\right)^{2}\left(\theta^{k}\right)^{2}}{2\left(1-\theta^{k}\right)} \leq Q\left(\|\boldsymbol{\mu}\|_{\infty}\right) .
$$

Therefore, it is sufficient to choose $\theta^{k}$ such that

$0<\theta^{k} \leq \frac{2 \sqrt{2 n \Gamma\left(\|\boldsymbol{\mu}\|_{\infty}\right)^{2}\left(-Q\left(\|\boldsymbol{\mu}\|_{\infty}\right)^{2}+Q\left(\|\boldsymbol{\mu}\|_{\infty}\right)\right)+Q\left(\|\boldsymbol{\mu}\|_{\infty}\right)^{2}}+2 Q\left(\|\boldsymbol{\mu}\|_{\infty}\right)^{2}-Q\left(\|\boldsymbol{\mu}\|_{\infty}\right)}{2 n \Gamma\left(\|\boldsymbol{\mu}\|_{\infty}\right)^{2}+4 Q\left(\|\boldsymbol{\mu}\|_{\infty}\right)^{2}}$.

It is interesting to note that this result does no longer depend on the choice of $\varphi$ only through the constant $T$.

Remark 3.3. We can compute an upper bound for $\theta^{k}$ by going to the limit, $\mu \downarrow 0$. Indeed, $\lim _{\mu \rightarrow 0} Q\left(\|\boldsymbol{\mu}\|_{\infty}\right)=1 / 2$ and $\lim _{\mu \rightarrow 0} \Gamma\left(\|\boldsymbol{\mu}\|_{\infty}\right)=1$, so any $\theta^{k}$ defined as in the previous theorem satisfies

$$
\theta^{k} \leq \frac{1}{\sqrt{2 n+1}}
$$

This upper bound is attained for $\varphi(t)=t$. 


\subsection{Complexity Analysis of the Full Newton Step IPM}

The complexity of this algorithm is obtained by the extension of a classical lemma.

Lemma 3.8. Let $\theta^{0}$ be such that $0<\theta^{0} \leq \theta^{k} \forall k \in \mathbb{N}$. The $\varphi$-FN-IPM for monotone LCP described in Algorithm 2 provides an $\epsilon$-solution $(z, s)$, which satisfies $\sum_{i=1}^{n} \varphi\left(z_{i} s_{i}\right) \leq n \epsilon$ after at most

$$
\left[\frac{1}{\theta^{0}} \log \left(\frac{\sum_{i=1}^{n} \varphi\left(z_{i}^{0} s_{i}^{0}\right)}{\epsilon}\right)\right]
$$

iterations.

Proof. Similar to the proof of [27.

The sequence $\left\{\theta^{k}\right\}$ is given by Theorem 3.4 and so our main theorem follows.

Theorem 3.5. Let $\boldsymbol{\mu}^{0}=z^{0} s^{0}$. Algorithm 2, with the sequence of update parameter $\theta^{k}$ described above, guarantees strict feasibility of the iterates and quadratic convergence of the proximity measure. Moreover, it provides an $\epsilon$-solution $(z, s)$, which satisfies $\sum_{i=1}^{n} \varphi\left(z_{i} s_{i}\right) \leq n \epsilon$ after at most

$$
\mathcal{O}\left(\sqrt{n} \log \left(\frac{n}{\epsilon}\right)\right)
$$

iterations.

\section{Numerics}

In this section, we provide insight on the behaviour of Algorithm 2 by running several computational tests.

\subsection{Implementation details}

The sequence of update parameters is given by Theorem 3.4 and we consider the functions $\varphi$ given in Table 1 . As pointed out earlier, the sequence of update parameters from Theorem 3.4 only relies on the constant $T$. Given a function $\varphi$, for any $\alpha$ positive $\alpha \varphi$ satisfies also all the condition from Section 3 . So that, it holds

$$
T_{\alpha \varphi}=-\frac{\varphi^{\prime \prime}(0)}{\alpha \varphi^{\prime}(0)}
$$

Therefore for any function $\varphi$, we can control the parameter $T$ by choosing the desired value of $\alpha$.

In Remark 3.2, we point out the fact that the algorithm must start with a $\boldsymbol{\mu}^{0}$ sufficiently small, that is $\boldsymbol{\mu}^{0} \leq \mu^{*}$. We compute $\mu^{*} \in \mathbb{R}_{+}$as a solution of

$$
0=a-\Gamma\left(\|\boldsymbol{\mu}\|_{\infty}\right)\left(\sqrt{2}+(13+2 \sqrt{6}) T\|\boldsymbol{\mu}\|_{\infty}\right),
$$




\begin{tabular}{|c|c|c|c|c|c|}
\hline Name & $\varphi(t)$ & $\varphi^{\prime}(t)$ & $\varphi^{\prime \prime}(t)$ & $\varphi^{-1}(t)$ & $T$ \\
\hline Id. & $\mathrm{t}$ & 1 & 0 & $\mathrm{t}$ & 0 \\
$\sqrt{ }$ & $\sqrt{t+1}-\sqrt{1}$ & $\frac{1}{2 \sqrt{t+1}}$ & $-\frac{1}{4(t+1)^{3 / 2}}$ & $t^{2}+2 t$ & 1 \\
$\log$ & $\log (1+t)$ & $\frac{1}{1+t}$ & $-\frac{1}{2(1+t)^{2}}$ & $\exp (t)-1$ & $\frac{1}{2}$ \\
$\theta^{1}$ & $\frac{t}{t+1}$ & $\frac{1}{(t+1)^{2}}$ & $-\frac{1}{2(t+1)^{3}}$ & $\frac{t}{1-t}$ & $\frac{1}{2}$ \\
\hline
\end{tabular}

Table 1: $\varphi$-functions used in the computational tests

where $a$ is chosen strictly greater than $\sqrt{2}$ and smaller than 2 , for instance $a=1.49$. We use the fsolve command from MATLAB. Given $\bar{z}^{0}, \bar{s}^{0}, \overline{\boldsymbol{\mu}}^{0} \in \mathbb{R}_{++}^{3 n}$ such that $\bar{z}^{0} \bar{s}^{0}=\overline{\boldsymbol{\mu}}^{0}$ and $\bar{s}^{0}=M \bar{z}^{0}+\bar{q}$, an initial point satisfying $\boldsymbol{\mu}^{0} \leq e \mu^{*}$, $s^{0}=M z^{0}+q$ and $\sum_{i=1}^{n} \varphi\left(z_{i}^{0}, s_{i}^{0}\right)=\sum_{i=1}^{n} \boldsymbol{\mu}_{i}^{0}$ can be constructed in the following way

$$
\boldsymbol{\mu}^{0}:=\boldsymbol{e} \mu^{*}, \sigma:=\sqrt{\frac{\varphi^{-1}\left(\boldsymbol{e} \mu^{*}\right)}{\overline{\boldsymbol{\mu}}^{0}}} z^{0}:=\bar{z}^{0} \sigma, s^{0}:=\bar{s}^{0} \sigma, q:=\bar{q} \sigma .
$$

At the end of the algorithm, for $k=k_{f}$, we get an approximate solution of (LCP by computing the reverse scaling :

$$
\left(z^{*}, s^{*}\right)=\frac{\left(z^{k_{f}}, s^{k_{f}}\right)}{\sigma}
$$

Notice that such a point $\bar{z}^{0}, \bar{s}^{0}$ can always be constructed using the self-embedding technique from [19].

Remark 4.1. One essential feature of the method presented in this paper is that for concave, increasing functions $\varphi$, the points can go further than the $\mu$-center expected. Indeed, considering that $\varphi$ is concave implies

$$
\boldsymbol{\varphi}\left(z^{+} s^{+}\right) \leq \boldsymbol{\varphi}(z s)+\varphi^{\prime}(z s)(z \Delta s+s \Delta z)+\varphi^{\prime}(z s) \Delta s \Delta z .
$$

Since $(\Delta z, \Delta s)$ is a solution of $(3)$, then

$$
\frac{\varphi\left(z^{+} s^{+}\right)-\boldsymbol{\mu}}{\varphi^{\prime}(z s)} \leq \Delta s \Delta z
$$

Therefore $\varphi\left(z^{+} s^{+}\right)$can be smaller than $\mu$. Taking into account the case, where $M$ is skew-symmetric. Then, $\Delta z^{T} \Delta s=0$, so

$$
\sum_{i=1}^{n} \frac{\varphi\left(z_{i}^{+} s_{i}^{+}\right)-\boldsymbol{\mu}_{i}}{\varphi^{\prime}\left(z_{i} s_{i}\right)} \leq 0
$$

whereas for $\varphi(t)=t$ we only have

$$
\sum_{i=1}^{n} \varphi\left(z_{i}^{+} s_{i}^{+}\right)=\left(z^{+}\right)^{T} s^{+}=\sum_{i=1}^{n} \boldsymbol{\mu}_{i}
$$

So, our method may behave better than the classical method in an algorithm that takes advantage of this property. 


\begin{tabular}{|c|c|c|c|c|}
\hline Instance & Id. & $\theta^{1}$ & $\log$ & $\sqrt{ }$ \\
\hline ADLITTLE & 135 & 135 & 135 & 135 \\
AFIRO & 85 & 85 & 85 & 85 \\
BEACONFD & 210 & 210 & 210 & 210 \\
BOEING2 & 210 & 210 & 210 & 210 \\
BLEND & 133 & 133 & 133 & 133 \\
GROW7 & 204 & 204 & 204 & 204 \\
ISRAEL & 215 & 215 & 215 & 215 \\
KB2 & 102 & 102 & 102 & 102 \\
RECIPELP & 166 & 166 & 166 & 166 \\
SC50A & 109 & 109 & 109 & 109 \\
SC50B & 109 & 109 & 109 & 109 \\
SC105 & 159 & 159 & 159 & 159 \\
SCAGR7 & 172 & 172 & 172 & 172 \\
SHARE1B & 186 & 186 & 186 & 186 \\
SHARE2B & 156 & 156 & 156 & 156 \\
STOCFOR1 & 163 & 163 & 163 & 163 \\
\hline
\end{tabular}

Table 2: Number of iterations to reach $(z)^{T} s \leq \epsilon=10^{-3}$

\subsection{Computational results}

The numerical tests are run on a small selection of LPs from the NETLIB repository 1 . The datasets and the number of iterations until convergence are detailed in Table 2 for every $\varphi$-function. We observe that on every instance, our method converges in the same number of iterations than the classical full step method $(\varphi(t)=t)$. Therefore, the experiments show the validity of our approach. Notice that for the three choices of function $\varphi$ the results are identical. This is not surprising, since the sequences of parameters $\left\{\theta^{k}\right\}$ and $\left\{\boldsymbol{\mu}^{k}\right\}$ depend on $T$ which is not really different for these 3 functions.

\section{Concluding remarks}

The method presented in this article shows a generalization of the FN-IPM with polynomial upper bound complexity for monotone LCPs considering a general family of smooth increasing concave functions. Further research may extend this result to a more general family of LCPs such that $P_{*}(\kappa)$-LCPs or $P_{0}$-LCPs with bounded level sets.

Recent developments by Roos in [26, consider an infeasible IPM with full Newton step using only one feasibility step. More investigations regarding the method presented in this paper could extend the results in [26.

We provide computational experiments to confirm the validity of our approach. Further investigations on more sophisticated methods may get the best

\footnotetext{
${ }^{1}$ http://www.netlib.org/lp/
} 
out of this new direction. Indeed, as stated in Remark 4.1 we expect an improved behavior by introducing a function $\varphi$ other than identity in an algorithm where the updates of $\boldsymbol{\mu}$ depends on the value of the iterates. In particular, we are planning to study a predictor-corrector implementation of this approach.

\section{References}

[1] M. Achache. A new primal-dual path-following method for convex quadratic programming. Computational \&3 Applied Mathematics, 25(1):97$110,2006$.

[2] M. Achache. Complexity analysis and numerical implementation of a shortstep primal-dual algorithm for linear complementarity problems. Applied Mathematics and computation, 216(7):1889-1895, 2010.

[3] M. Anitescu, G. Lesaja, and F. A. Potra. Equivaence between different formulations of the linear complementarity promblem. Optimization Methods and Software, 7(3-4):265-290, 1997.

[4] S. Asadi and H. Mansouri. Polynomial interior-point algorithm for $P_{*}(\kappa)$ horizontal linear complementarity problems. Numerical Algorithms, 63(2):385-398, 2013.

[5] S. Asadi and H. Mansouri. A new full-newton step $\mathrm{O}(\mathrm{n})$ infeasible interiorpoint algorithm for $P_{*}(\kappa)$-horizontal linear complementarity problems. The Computer Science Journal of Moldova, 22(1):37-61, 2014.

[6] S. Asadi, H. Mansouri, and Z. Darvay. An infeasible interior-point method with improved centering steps for monotone linear complementarity problems. Asian-European Journal of Mathematics, 8(03):1550037, 2015.

[7] Y.Q. Bai, G. Lesaja, C. Roos, G.Q. Wang, and M. El Ghami. A class of large-update and small-update primal-dual interior-point algorithms for linear optimization. Journal of Optimization Theory and Applications, 138(3):341-359, 2008.

[8] S.J. Chung. A note on the complexity of LCP: the LCP is strongly npcomplete. Technical Reprot, pages 79-2, 1979.

[9] R. W. Cottle. A field guide to the matrix classes found in the literature of the linear complementarity problem. Journal of Global Optimization, 46(4):571-580, 2010.

[10] R. W. Cottle, J.-S. Pang, and R. E. Stone. The linear complementarity problem, volume 60. Siam, 2009.

[11] Z. Darvay. New interior-point algorithms in linear programming. Advanced Modelling and Optimization, 5(1):51-92, 2003. 
[12] E. de Klerk and M. Negy. On the complexity of computing the handicap of a sufficient matrix. Mathematical programming, 129(2):383-402, 2011.

[13] M. El Ghami. New primal-dual interior-point methods based on kernel functions. PhD thesis, 2005.

[14] G. Gu. Full-step interior-point methods for symmetric optimization. $\mathrm{PhD}$ thesis, 2009.

[15] M. Haddou and P. Maheux. Smoothing methods for nonlinear complementarity problems. Journal of Optimization Theory and Applications, 2014.

[16] T. Illés, C. Roos, and T. Terlaky. Polynomial affine-scaling algorithms for $P_{*}(\kappa)$ linear complementary problems. In Recent Advances in Optimization, pages 119-137. Springer, 1997.

[17] F. Jarre and M. A. Saunders. An adaptive primal-dual method for linear programming. Technical report, DTIC Document, 1991.

[18] N. Karmarkar. A new polynomial-time algorithm for linear programming. In Proceedings of the sixteenth annual ACM symposium on Theory of computing, pages 302-311. ACM, 1984.

[19] M. Kojima, N. Megiddo, T. Noma, and A. Yoshise. A unified approach to interior point algorithms for linear complementarity problems, volume 538. Springer Science \& Business Media, 1991.

[20] G. Lesaja and C. Roos. Unified analysis of kernel-based interior-point methods for $P_{*}(\kappa)$-linear complementarity problems. SIAM Journal on Optimization, 20(6):3014-3039, 2010.

[21] H. Mansouri. Full-Newton step interior-point methods for conic optimization. PhD thesis, 2008.

[22] H. Mansouri, M. Zangiabadi, and M. Pirhaji. A full-newton step O(n) infeasible-interior-point algorithm for linear complementarity problems. Nonlinear Analysis: Real World Applications, 12(1):545-561, 2011.

[23] J. Peng, C. Roos, and T. Terlaky. Self-Regularity: A new paradigm for Primal-Dual interior-point algorithms. Princeton University Press, 2009.

[24] J. Renegar. A mathematical view of interior-point methods in convex optimization, volume 3. Siam, 2001.

[25] C. Roos. A full-newton step $\mathrm{O}(\mathrm{n})$ infeasible interior-point algorithm for linear optimization. SIAM Journal on Optimization, 16(4):1110-1136, 2006.

[26] C. Roos. An improved and simplified full-newton step $\mathrm{O}(\mathrm{n})$ infeasible interior-point method for linear optimization. SIAM Journal on Optimization, 25(1):102-114, 2015. 
[27] C. Roos, T. Terlaky, and J.-Ph. Vial. Interior point methods for linear optimization. Springer Science \& Business Media, 2006.

[28] T. Terlaky. Interior point methods of mathematical programming, volume 5. Springer Science \& Business Media, 2013.

[29] G.Q. Wang, X.J. Fan, D.T. Zhu, and D.Z. Wang. New complexity analysis of a full-newton step feasible interior-point algorithm for $P_{*}(\kappa)$-lcp. Optimization Letters, 9(6):1105-1119, 2015.

[30] Y. Ye. Interior point algorithms: theory and analysis, volume 44. John Wiley \& Sons, 2011. 